\title{
ON THE ENTROPY OF CERTAIN CLASSES OF SKEW-PRODUCT TRANSFORMATIONS
}

\section{NEWTON}

1. Introduction. For the theory of Lebesgue spaces, skew-product transformations and entropy see [5], [4] and [6]. Let $X$ and $Y$ be Lebesgue spaces with measures $\mu$ and $\nu$ and let $M=X \times Y$ be the direct product space with the direct product measure $\mu \times \nu$. Let $S$ be an automorphism of the space $X$ and let $\left\{T_{x}\right\}$ be a family of automorphisms of the space $Y$, define $U(x, y)=\left(S x, T_{x} y\right)$. Under certain measurability conditions on the family $\left\{T_{x}\right\} U$ is an automorphism of the space $M . U$ is called the skew-product with base $S$ and fibre $T_{x}$. The entropy of such a skew-product has been calculated [1], [4], but the formulae given are not in general amenable to calculation.

We will consider two special types of skew-product:

1. Let $T$ be an automorphism of $Y$ and let $k(x)$ be an integer valued function on $X$ such that $k(x)>0$ and $\int_{X} k(x) d \mu<\infty$. The first class of skew-products are those for which $T_{x}=T^{k(x)}$.

2. Let $\psi_{t}$ be a measurable measure-preserving flow on $Y$ and let $f(x)$ be a real-valued function on $X$ such that $f(x) \geqq \tau>0$, for some constant $\tau$, and $\int_{X} f(x) d \mu<\infty$. The second class of skew-products are those of the form $T_{x}=\psi_{f(x)}$.

In both these special cases we will show that $h(U)=h(S)$ $+\int_{X} h\left(T_{x}\right) d \mu$. We note that the first class is not contained in the second since the automorphism $T$ may not be embeddable in a flow.

2. Induced automorphisms and special flows. Let $T$ be an automorphism of a Lebesgue space $M$ with measure $\mu$. If $X \subset M$ and $\mathrm{U}_{n=0}^{\infty} T^{n} X=M$, then $T$ induces in the Lebesgue space $X$, with measure $\mu_{X}(A)=\mu(A)(\mu(X))^{-1}, A \subset X$, the induced automorphism $T^{\prime}$ given by $T^{\prime} x=T^{k(x)} x(x \in X)$ where $k(x)$ is the smallest positive integer $l$ such that $T^{l} x \in X$. Abramov [2] has shown that $h(T)=h\left(T^{\prime}\right) \mu(X)$.

Let us now consider the special flow built on an automorphism $S$ of a Lebesgue space $M$ under a function $f . f$ is a real-valued function on $M$ such that

(i) $f(x) \geqq \tau>0$ for $x \in M$,

(ii) $\int_{M} f(x) d \mu<\infty$.

Let $V$ be the subset of $M \times R, R$ is real line, defined by $(x, u) \in V$ if $0 \leqq u<f(x)$. We define a group of automorphisms on $V$ as follows; for $t<\tau$

Received by the editors November 21, 1967. 


$$
\begin{aligned}
S_{t}(x, u) & =(x, u+t) \quad \text { if } t<f(x)-u, \\
& =(S x, t+u-f(x)) \quad \text { if } t \geqq f(x)-u,
\end{aligned}
$$

for the remaining values of $t$ the automorphisms $\left\{S_{t}\right\}$ can be defined so that $\left\{S_{t}\right\}$ is a group. For an explicit formula for $S_{t}$ see [6]. Abramov [3] has shown that $h\left(S_{t}\right)=|t| h(S)\left(\int_{M} f(x) d \mu\right)^{-1}$. We note that the entropy of an automorphism is always calculated with respect to a normalised measure space.

\section{Main theorems.}

THEOREM 1. Let $U$ on $X \times Y$ with measure $\mu \times \nu$ be defined by $U(x, y)$ $=\left(S x, T^{k(x)} y\right)$ where $\infty>k(x)>0, k(x)$ is integer valued and $\int_{X} k(x) d \mu$ $<\infty$. Then

$$
h(U)=h(S)+h(T) \int_{X} k(x) d \mu=h(S)+\int_{X} h\left(T^{k(x)}\right) d \mu .
$$

Proof. Let $Z$ denote the set of positive integers $Z=\{1,2, \cdots\}$ and let $\eta$ be the measure on $Z$ which assigns measure 1 to each point. Let $V$ be the subset of $X \times Y \times Z$ defined by $(x, y, i) \in V$ if $i \leqq k(x)$. Note that $V=V^{\prime} \times Y$ where $V^{\prime}$ is the subset of $X \times Z$ defined by $(x, i) \in V^{\prime}$ if $i \leqq k(x)$. It is easily seen that

$$
\mu \times \nu \times \eta(V)=\mu \times \eta\left(V^{\prime}\right)=\int_{X} k(x) d \mu .
$$

Let us consider $V$ as a Lebesgue space with the measure normalised, i.e., if $A \subset V$ then

$$
\mu^{\prime}(A)=\mu \times \nu \times \eta(A) \cdot\left(\int_{X} k(x) d \mu\right)^{-1} .
$$

Define an automorphism $\phi$ on $V$ by

$$
\begin{aligned}
\phi(x, y, i) & =(x, T y, i+1) \quad \text { if } i<k(x), \\
& =(S x, T y, 1) \quad \text { if } i=k(x) .
\end{aligned}
$$

Then $\phi=T \times \psi$ where $\psi$ is the automorphism on $V^{\prime}$ defined by

$$
\begin{aligned}
\psi(x, i) & =(x, i+1) \quad \text { if } i<k(x) . \\
& =(S x, 1) \quad \text { if } i=k(x) .
\end{aligned}
$$

It is clear that $U$ is the automorphism induced by $\phi$ on the set $X \times Y \times\{1\}$. Thus we have

$$
h(U)=h(\phi)\left(\mu^{\prime}(X \times Y \times\{1\})\right)^{-1}=\int_{X} k(x) d \mu h(\phi) .
$$


Since $\phi=T \times \psi$ it follows that $h(\phi)=h(T)+h(\psi)$ and it remains only to calculate $h(\psi)$. It is clear that $S$ is the automorphism induced by $\psi$ on the set $X \times\{1\}$; thus $h(\psi)=h(S) \cdot\left(\int_{X} k(x) d \mu\right)^{-1}$. Finally

$$
\begin{aligned}
h(U) & =h(S)+\int_{X} k(x) d \mu \cdot h(T) \\
& =h(S)+\int_{X} h\left(T^{k(x)}\right) d \mu .
\end{aligned}
$$

TheOREM 2. Let $U$ on $X \times Y$ with measure $\mu \times \nu$ be defined by $U(x, y)$ $=\left(S x, \psi_{f(x)} y\right)$ where $f(x)$ is a real-valued function on $X, \infty>f(x) \geqq \tau>0$ such that $\int_{X} f(x) d \mu<\infty$. Then

$$
h(U)=h(S)+\int_{X} f(x) d \mu h\left(\psi_{1}\right)=h(S)+\int_{X} h\left(\psi_{f(x)}\right) d \mu .
$$

Proof. Let $R$ be the set of real numbers with the usual Lebesgue measure. Let $A$ be the subset of $X \times Y \times R$ defined by $(x, y, u) \in A$ if $0 \leqq u<f(x)$ we note that $A=A^{\prime} \times Y$ where $A^{\prime}$ is the subset of $X \times R$ defined by $(x, u) \in A^{\prime}$ if $0 \leqq u<f(x)$. We consider the following flows defined on $A, A$ and $A^{\prime}$ respectively, all are defined for $t<\tau$ and extended by group property,

$$
\begin{aligned}
\phi_{t}(x, y, u) & =\left(x, \psi_{t} y, u+t\right) \quad \text { if } t<f(x)-u, \\
& =\left(S x, \psi_{t} y, u+t-f(x)\right) \quad \text { if } t \geqq f(x)-u ; \\
\phi_{t}^{\prime}(x, y, u) & =(x, y, u+t) \quad \text { if } t<f(x)-u, \\
& =\left(S x, \psi_{f(x)} y, u+t-f(x)\right) \quad \text { if } t \geqq f(x)-u ; \\
\phi_{t}^{\prime \prime}(x, u) & =(x, u+t) \quad \text { if } t<f(x)-u, \\
& =(S x, u+t-f(x)) \quad \text { if } t \geqq f(x)-u .
\end{aligned}
$$

It is easy to see that $\phi_{t}$ is a direct product of the flow $\psi_{t}$ on $Y$ with the flow $\phi_{t}^{\prime \prime}$ on $A^{\prime}$. It is also clear that $\phi_{t}^{\prime}$ and $\phi_{t}^{\prime \prime}$ are in the form of special flows built over the automorphisms $U$ and $S$ respectively and with the same function $f(x)$, regarded firstly as a function of two variables $f(x, y) \equiv f(x)$ and secondly just as a function of one variable. Using Abramov's formula

$$
\begin{aligned}
& h\left(\phi_{t}^{\prime}\right)=|t| h(U)\left(\int_{X} f(x) d \mu\right)^{-1}, \\
& h\left(\phi_{t}^{\prime \prime}\right)=|t| h(S)\left(\int_{X} f(x) d \mu\right)^{-1} .
\end{aligned}
$$

Also $h\left(\phi_{t}\right)=h\left(\psi_{t}\right)+h\left(\phi_{t}^{\prime \prime}\right)$. We will exhibit an isomorphism $U$ of $A$ 
to itself which has the property that $U^{-1} \phi_{t} U=\phi_{t}^{\prime}$ for all $t$. Define

$$
U(x, y, u)=\left(x, \psi_{u} y, u\right) \text {; }
$$

then, it is easily checked that $U$ satisfies the required property. Thus $h\left(\phi_{t}\right)=h\left(\phi_{t}^{\prime}\right)$. Gathering together the formulae we have derived we obtain

$$
h\left(\phi_{t}\right)=|t| h(U)\left(\int_{X} f(x) d \mu\right)^{-1}=h\left(\psi_{t}\right)+|t| h(S)\left(\int_{X} f(x) d \mu\right)^{-1}
$$

or, in other words,

$$
\begin{aligned}
h(U) & =h(S)+h\left(\psi_{t}\right)|t|^{-1} \int_{X} f(x) d \mu \\
& =h(S)+h\left(\psi_{1}\right) \int_{X} f(x) d \mu,
\end{aligned}
$$

since $h\left(\psi_{t}\right)=|t| h\left(\psi_{1}\right)$.

The proof of Theorem 1 depends on the restriction that $0<k(x)$ and a natural question is to ask if the theorem is true when we let $k(x)$ take both positive and negative values. The following partial result was pointed out to us by W. Parry.

ThEOREM 3. Let $h(x)$ be an integer valued function on $X$ with the following properties

(i) $\int_{X} h(x) d \mu<\infty$.

(ii) There exists an integer-valued function $k(x)$ and an integrable integer-valued function $l(x)$ such that $h(x)=k(x)-l(x)+l(S x)$ and $0<k(x)<\infty$.

Then, if $U(x, y)=\left(S x, T^{h(x)} y\right), h(U)=h(S)+h(T) \int_{x} h(x) d \mu$.

We remark that it is possible to find functions $h(x)$ which take both positive and negative values and in such cases

$$
h(T) \int_{X} h(x) d \mu \neq \int_{X} h\left(T^{h(x)}\right) d \mu .
$$

Proof. Let $\phi(x, y)=\left(x, T^{l(x)} y\right)$; then

$$
\begin{aligned}
\phi^{-1} U \phi(x, y) & =\left(S x, T^{-l(S x)} T^{h(x)} T^{l(x)} y\right) \\
& =\left(S x, T^{k(x)} y\right) .
\end{aligned}
$$

By Theorem 1,

$$
h(U)=h\left(\phi^{-1} U \phi\right)=h(S)+h(T) \int_{X} k(x) d \mu .
$$


But $h(x)=k(x)-l(x)+l(S x)$; therefore, since $S$ preserves the measure $\mu$,

$$
\int_{\boldsymbol{X}} h(x) d \mu=\int_{\boldsymbol{X}} k(x) d \mu .
$$

Thus $h(U)=h(S)+h(T) \int_{x} h(x) d \mu$. A theorem of a similar type will also extend Theorem 2.

\section{REFERENCES}

1. R. Adler, $A$ note on the entropy of skew product transformations, Proc. Amer. Math. Soc. 4 (1963), 665-669.

2. L. M. Abramov, The entropy of a derived automorphism, Dokl. Akad. Nauk SSSR 128 (1959), 647-650; Amer. Math. Soc. Transl. (2) 49 (1966), 162-166.

3. - The entropy of a flow, Dokl. Akad. Nauk SSSR 128 (1959), 873-875; Amer. Math. Soc. Transl. (2) 49 (1966), 167-170.

4. L. M. Abramov and V. A. Rohlin, The entropy of a skew product of measure preserving transformations, Vestnik Leningrad Univ. 7 (1962), 5-13; Amer. Math. Soc. Transl. (2) 48 (1965), 255-265.

5. V. A. Rohlin, On the fundamental ideas of measure theory, Mat. Sb. 25 (1949), 107-150; Amer. Math. Soc. Transl. (1) 10 (1962), 1-54.

6. - New progress in the theory of transformations with invariant measure, Uspehi Mat. Nauk 15 (1960), no. 4 (94), 3-26=Russian Math. Surveys 15 (1960), no. 4, 1-22.

University of Sussex, Falmer, Brighton 